\title{
The relationship between infertility family stigma, self-stigma and psychological well-being in female first-visit patients at a reproductive medicine center in Taiwan
}

\begin{abstract}
BACKGROUND
Steeped in Chinese culture which considers continuing the family lineage highly important, infertility stigma endorsed by others and oneself can both negatively impact psychological well-being in women with infertility in Taiwan. The aim of the present study was to find out whether family stigma attached to infertility has a direct effect on psychological well-being, or whether it is only when family stigma is internalized into self-stigma that psychological well-being is affected.
\end{abstract}

\section{PARTICIPANTS AND PROCEDURE}

The present study had a cross-sectional design, approved by an institutional review board in Taiwan. 245 female participants with infertility completed measures on infertility stigma, self-esteem, and positive and negative affect. The main question of this study was analyzed by conducting structural equation modeling.
RESULTS

The present study found that none of the direct effects of family stigma on self-esteem, negative affect, or positive affect were significant, while the indirect effects of family stigma on the three psychological well-being indexes through the mediator of self-stigma were significant.

\section{CONCLUSIONS}

Self-stigma fully mediates the effect of family stigma on self-esteem, positive affect, and negative affect in women with infertility. Collectively, stigma endorsed by others does not necessarily lead to negative psychological wellbeing. The negative influence comes from internalizing public/family stigma into self-stigma.

KEY WORDS

family stigma; infertility; public stigma; self-stigma

ORgANIZATION - 1: Department of Psychology, National Chengchi University, Taipei, Taiwan · 2: Department of Psychology, Soochow University, Taipei, Taiwan · 3: Xiamen EMBO Hospital, Xiamen City, Fujian, China • 4: Department of Educational Psychology and Counseling, National Pingtung University, Pingtung, Taiwan .

5: Research Center for Mind, Brain and Learning, National Chengchi University, Taipei, Taiwan AUthors' Contributions - A: Study design - B: Data collection - C: Statistical analysis - D: Data interpretation . E: Manuscript preparation · F: Literature search · G: Funds collection Corresponding Author - Wen-Yau Hsu, Ph.D., Department of Psychology, National Chengchi University, 64, Sec. 2, ZhiNan Road, Wenshan District, Taipei 11605, Taiwan, e-mail: hsu@nccu.edu.tw 


\section{BACKGROUND}

Previous studies have shown that patients with infertility face psychosocial problems and that their emotions (Abbey et al., 1991; Klemetti et al., 2010; Tiu et al., 2018) and coping behaviors (Hsu \& Kuo, 2002) are negatively affected. The cost of assisted reproductive technology (ART) treatment is also a burden and challenge for many couples with infertility in Taiwan (Cheng et al., 2018). In Chinese culture, to fulfill filial piety, married couples have the duty to support and respect one's parents, and most importantly to continue the family lineage (Logan et al., 2019). Going through the infertility experience is a stressful event for Chinese couples. To add to this adversity, people tend to consider childlessness as the fault of women in Chinese culture (Li, 2008; Tiu et al., 2018). In ancient China, inability to reproduce an heir is the most unfilial act and a reason for divorce initiated by the husbands. To fulfil the demands of the husband's family and maintain the stability of marriage, some women invest all their energy and fortune to bear children. Childbearing also serves as a way to womanhood (Yao et al., 2018). Failing to become a mother may evoke feelings of inadequacy and incompleteness in women, which can further cause a loss of one's self- and social identity (Yao et al., 2018). The rate and level of feeling ashamed and stigmatized because of infertility are higher among wives than husbands in couples (Ergin et al., 2018; Lau et al., 2008; Lin et al., 2006; Slade et al., 2007; Tiu et al., 2018; Wu, 2002). Therefore, the present study focused on stigma in women with infertility.

The way that stigmatized individuals understand and interpret the stigma endorsed by society is crucial in deciding to what extent the stigma affects individuals' self-esteem and emotion (Corrigan \& Watson, 2002; Corrigan et al., 2011). Society establishes normative expectations and demands of what the individual ought to be. Stigma occurs when there is a discrepancy between virtual social identity and actual social identity (Goffman, 1963). However, having knowledge of a set of stigmata, stereotypes, and social norms does not mean the individual agrees with them (Jussim et al., 1995). In a prior study, only when patients agreed with stigma endorsed by others, and internalized this stigma into self-stigma, were negative effects on psychological well-being observed (Corrigan et al., 2011).

Based on the perspectives mentioned above, there are two possibilities with regard to how infertility stigma may have a negative effect on women with infertility. One is living in a culture that strongly values filial piety, as in China. Women with infertility may perceive stigma from people around them because of their inability to bear children. This kind of stigma is referred to as public stigma (Fu et al., 2015), which can bring the feeling of isolation, being gossiped about or insulted for infertility, perception of social exclusion, and feeling like a second-class person in women with infertility (Ergin et al., 2018; Fu et al., 2015; Huang et al., 2019). The public stigma also has a negative impact when women with infertility engage in assisted reproductive technology treatment. In Chinese culture, reproductive capacity is associated with face, reputation and self-esteem. To save face, couples with infertility may conceal their infertility, limiting the accessibility to family support during the treatment (Yao et al., 2018). It is expected that public stigma would correlate with negative psychological well-being in women with infertility in such an environment (Fu et al., 2015; Yao et al., 2018). However, according to Corrigan et al. (2011), only when individuals internalize public stigma into self-stigma would it bring harm to psychological well-being; therefore, the other possibility is that public stigma has a negative impact through the psychological process of internalization. For instance, women in Chinese culture tend to agree with the importance of childbearing, internalizing public stigma into self-stigma. Therefore, infertile women may blame themselves for infertility, feeling guilty and emotionally suffocated by being unable to meet social norms and family expectations (Yao et al., 2018). The aim of the present study was to investigate both possibilities in the relationship between infertility stigma and psychological well-being in women with infertility.

Currently, in Taiwan there is controversy regarding the importance and value of filial piety (Yeh, 2003). On the one hand, filial piety is still a valued virtue in Taiwan, emphasizing that supporting, respecting, and repaying parents' kindness can lead to less conflict and problems in the family. On the other hand, there is a growing clamor for pursuing self-fulfillment, and the belief that prioritizing parents or family over oneself will obstruct the development of autonomy and self-actualization. As a result of modernization and the influence of Western culture, people in Taiwan may not totally agree with or internalize infertility stigma; therefore, the effect of filial piety on Taiwanese people may not be as strong as in China. Whether public stigma attached to infertility has a direct effect on psychological well-being or whether it is only when women with infertility internalize the stigma endorsed by others that psychological well-being is affected is unclear with regard to Taiwanese women with infertility.

Based on the World Population Review (2019), "Taiwan maintains one of the world's lowest birth rates" (para. 13), showing a trend towards fewer children. A couple with no children is not necessarily discriminated against by society like in the past, 40-50 years ago. However, continuing the family lineage is still valued by the oldest members in a family, who either intentionally or unintentionally tell the couple to bear children (Wu, 2002). Some literature indicates that being misunderstood and blamed for infertility by one's family is especially stressful for
Infertility stigma in Taiwan 
Yi-Tung Lin, Ashley W. Wang, Shirley Wei, Jia-Sin Hong, Wen-Yau Hsu women with infertility concerns in Taiwan (Lin et al., 2006; Yang et al., 2007). Therefore, instead of investigating the effects of public stigma, which is the stigma endorsed by the general community, the present study focused on family stigma, which is stigma endorsed by the family members of the infertile couple. The example of family stigma against infertility can be the experiences that family members do not take care of them as much as before, or being overworked and taunted by the family because of the infertility (Fu et al., 2015).

To investigate the mechanism of how family stigma and self-stigma had an impact on women with infertility in Taiwan, we examined the direct and indirect effects of family stigma on psychological well-being. We hypothesized that self-stigma would act as a mediator in the relationship between family stigma and psychological well-being. Based on the perspective of Corrigan and Watson (2002), stigma can have an effect on one's self-esteem and emotions. Therefore, the present study examined self-esteem, positive affect, and negative affect as indicators of psychological well-being. To test the hypothesized mediational relationship, structural equation modeling (SEM) was used to examine

\section{Table 1}

Results of exploratory factor analysis and Cronbach's alphas for two-factor solution

\begin{tabular}{lcc}
\hline Items & \multicolumn{2}{c}{ Factor loadings } \\
\cline { 2 - 3 } & $\begin{array}{c}\text { Family } \\
\text { stigma }\end{array}$ & $\begin{array}{c}\text { Self- } \\
\text { stigma }\end{array}$ \\
\hline $\begin{array}{l}\text { 1. Having no child, I feel that } \\
\text { the family members look } \\
\text { down on me. }\end{array}$ & .87 & .00 \\
$\begin{array}{l}\text { 2. Having no child, I feel that it } \\
\text { is difficult to get along with }\end{array}$ & .90 & .04 \\
my family. & & \\
3. Having no child, I feel further & .97 & -.07 \\
apart from my family. & & \\
$\begin{array}{l}\text { 4. Having no child, I have } \\
\text { a lower status in my family. }\end{array}$ & .92 & .01 \\
$\begin{array}{l}\text { 5. Having no child, I feel that } \\
\text { I am being attacked and }\end{array}$ & .78 & .05 \\
blamed personally. & & \\
$\begin{array}{l}\text { 6. Having no child, I blame } \\
\text { myself. }\end{array}$ & & \\
$\begin{array}{l}\text { 7. Having no child, I usually } \\
\text { encounter situations that } \\
\text { make me upset. }\end{array}$ & .03 & .94 \\
Eigenvalue \\
Cronbach's $\alpha$
\end{tabular}

nots Hospital Institutional Review Board. After introducing the study and obtaining informed consent from the participants, the researcher had the participants complete the questionnaire.

\section{INSTRUMENTS}

Infertility stigma. Based on previous studies regarding infertility stigma, and the constructs of self-stigma and family stigma, a 7-item Infertility Stigma Scale was developed via exploratory factor analysis (EFA) and confirmatory factor analysis (CFA). The modified model in CFA obtained indexes of goodness-of-fit as follows: $\chi^{2}=16.94, d f=9, \chi^{2} / d f<3$, NFI $=.98, \mathrm{NNFI}=.98$, $\mathrm{CFI}=.99$, GFI $=.96$, AGFI $=.89$, SRMR $=.04$, and RMSEA $=.085$, showing a good fit to the data. This Infertility Stigma Scale consists of two factors measuring the constructs of family stigma and self-stigma. There are 5 items regarding family stigma, with Cronbach's $\alpha=.93$. The self-stigma was measured by the other 2 items, with Cronbach's $\alpha=.83$. Items were rated on a 5-point Likert-type scale ranging from 1 (strongly disagree) to 5 (strongly agree). Higher scores reflect greater infertility stigma. The items and their factor loadings are shown in Table 1.

Self-esteem. The 6-item version of the Rosenberg Self-Esteem Scale (Abbey et al., 1991; Rosenberg, 1965) was used to assess self-esteem. Responses are 
made on a 5-point Likert-type scale with options ranging from 1 (strongly disagree) to 5 (strongly agree). The Cronbach's $\alpha$ was .70 in the present study.

Affect. Based on the Profile of Mood States (POMS) and the Infertility Specific Well-Being and Distress Scales (McNair et al., 1971; Stanton, 1991), the present study created a 6-item scale to assess positive affect consisting of items such as "optimistic" and "satisfied," and an 8-item scale to assess negative affect consisting of items such as "depressive" and "worried." Responses were made on a 5-point Likert-type scale with response options that ranged from not at all to extremely. The Cronbach's $\alpha$ was .88 for the Positive Affect Scale and .89 for the Negative Affect Scale.

\section{STATISTICAL ANALYSIS}

The data were analyzed with IBM SPSS Statistics 22 version and LISREL Student version. Only two percent of the participants had missing data on the scales assessing self-esteem and affect, which were substituted with median scores. Pearson's correlation coefficients were used to examine the relationships between stigma and psychological well-being (i.e., self-esteem, positive affect, and negative affect). Partial correlation coefficients were examined for the relationships between one kind of stigma and the psychological outcomes after controlling for the other kind of stigma. SEM was performed to test hypothesized mediation models. The indexes suggested by $\mathrm{Hu}$ and Bentler (1999) were used to assess the goodness-of-fit of the models.

\section{RESULTS}

\section{RELATIONSHIPS BETWEEN INFERTILITY STIGMA AND PSYCHOLOGICAL WELL-BEING}

As can be seen in Table 2, both family stigma and self-stigma were negatively associated with selfesteem and positive affect, and both were positively associated with negative affect, consistent with the expectations of the present study. Therefore, partial correlations were conducted to investigate the relationship between one kind of stigma and psychological well-being after controlling for the other kind of stigma. The results showed that after controlling for family stigma, self-stigma significantly negatively correlated with self-esteem, $r=-.26, p<.001$, and positive affect, $r=-.22, p<.001$, and significantly positively correlated with negative affect, $r=.33$, $p<.001$. When controlling for self-stigma, the correlation between family stigma and negative affect remained significant, $r=.20, p<.05$, while the other two correlations (i.e., with self-esteem and positive affect) were no longer significant.

\section{TEST OF THE MEDIATION MODEL}

The present study further investigated whether selfstigma acted as a mediator in the relationship between family stigma and psychological outcomes.

In the estimation of the partial mediation models of the three psychological outcomes, the results showed that the models fit the data. However, none of the direct effects of family stigma on self-esteem, negative affect, or positive affect were significant $(z=.03, .09$, -.02 , respectively, $p>.05$ ). Therefore, the direct effects were removed from the models, and SEM was conducted to estimate the full mediation models. The results showed that each model had an excellent fit with the data (see Table 3). The indirect effects of family stigma on the psychological outcomes of self-esteem, negative affect, and positive affect through the mediator of self-stigma were all significant $(z=-.20, .32$, -.20 , respectively, $p<.05$ ). Therefore, the relationship between family stigma and psychological well-being was fully mediated by self-stigma.

\section{DISCUSSION}

The present study found that self-stigma fully mediated the effect of family stigma on self-esteem, positive affect, and negative affect in women with infertility.

\section{Table 2}

Means, standard deviations, and correlation coefficients among variables

\begin{tabular}{lccccccc}
\hline Measure & $M$ & $S D$ & 1 & 2 & 3 & 4 & 5 \\
\hline 1. Self-esteem & 21.16 & 3.15 & - & $-.49^{* *}$ & $.46^{* *}$ & $-.18^{*}$ & $-.31^{* *}$ \\
2. Negative affect & 19.19 & 5.80 & $-57^{* *}$ & $.40^{* *}$ & $.47^{* *}$ \\
3. Positive affect & 18.97 & 4.21 & & $-.56^{* *}$ & - & $-.21^{* *}$ & $-.30^{* *}$ \\
4. Family stigma & 9.07 & 5.21 & & & - & $.53^{* *}$ \\
5. Self-stigma & 5.11 & 2.26 & & & & - \\
\hline
\end{tabular}

Note. ${ }^{*} p<.01$ (two-tailed); ${ }^{* *} p<.001$ (two-tailed). 
Table 3

Indexes of goodness-of-fit in the full mediation models

\begin{tabular}{lrcccccccc}
\hline & \multicolumn{1}{c}{$\chi^{2}$} & $d f$ & NFI & NNFI & CFI & GFI & AGFI & SRMR & RMSEA \\
\hline Self-esteem & 99.03 & 60 & .952 & .974 & .983 & .943 & .913 & .060 & .052 \\
Negative affect & 149.94 & 83 & .943 & .966 & .973 & .927 & .895 & .073 & .057 \\
Positive affect & 74.75 & 55 & .967 & .987 & .991 & .958 & .930 & .048 & .038 \\
\hline
\end{tabular}

Yi-Tung Lin, Ashley W. Wang, Shirley Wei, Jia-Sin Hong, Wen-Yau Hsu

Note. NFI - normed fit index; NNFI - non-normed fit index; CFI - comparative fit index; GFI - goodness-of-fit index; AGFI adjusted GFI; SRMR - standardized root-mean-square residual; RMSEA - root-mean-square error of approximation.

These results are similar to Vogel et al. (2007), who found that self-stigma fully mediated the effect of perceived stigma (public stigma) on college students' attitudes toward counseling and their willingness to seek help. Also, the results of the present study are consistent with the findings in patients with breast or prostate cancer (Else-Quest et al., 2009), and psychiatric patients (Corrigan et al., 2011).

According to Corrigan et al.'s (2011) progressive model of self-stigma, only when individuals are aware of associated stereotypes, agreeing with these stereotypes, applying the stereotypes to oneself, would it cause harm in one's self. The results of the present study showed that when women with infertility internalize the stigma into self-stigma, harm to oneself, higher negative affect and lower positive affect were observed, which echoed the progressive model of selfstigma (Corrigan et al., 2011).

The results of the present study are surprising in the context of Chinese culture. Traditionally, childbearing is a way to cultivate interpersonal relationships in the family, as well as to maintain social integrity and cohesion (Logan et al., 2019). In this collectivist culture, the demands and interests of society/ family take precedence over the desires and needs of individuals (Wagner III, 1995); people tend to adhere to social norms and attach greater importance to smooth and harmonious interpersonal relationships with society/family (Chang \& Holt, 1991; Tsui \& Farh, 1997). However, as Taiwan has become more industrialized and modernized, childlessness has become more voluntary (Poston, 1988). Indeed, people do not value the importance of continuing the family lineage as much as previous generations (Lee, 2009). The increased educational level and employment opportunities have also empowered women in Taiwan nowadays. Infertile women with higher socioeconomic status and education level tend to have better self-sufficiency and self-regulation of negative emotion (Logan et al., 2019; Jin et al., 2013). In an empirical study, women who were engaging in infertility treatment reported low levels of fertility-related stress in Taiwan (Cheng et al., 2018), which was inconsistent with other studies (Loke et al., 2012). This result might be associated with the lower self-stigma about infer- tility today in Taiwan. Based on Corrigan and Watson (2002), stereotypes and stigma do not necessarily lead to harmful self-identity, social identity, and decreased self-esteem. The negative influence comes from internalizing the public/family stigma, which is referred to as self-stigma.

However, internalizing stereotypes and stigma may not be the only way that one's psychological functioning can be affected. In the present study, after controlling for self-stigma the partial correlation between family stigma and negative affect was still significant, which is similar to results found in previous studies (Donkor \& Sandall, 2007; Fu et al., 2015; Slade et al., 2007). Additionally, Pescosolido and Martin (2015) pointed out that public stigma is a multi-dimensional construct, which includes social distance, traditional prejudice, exclusionary sentiments, and disclosure carryover. The Infertility Stigma Scale developed in the present study was based on the concept of filial piety in Chinese culture, which is similar to traditional prejudice. Based on Pescosolido and Martin's (2015) concept of multiple dimensions of public stigma, whether similar results would be found for traditional prejudice is an area for future research investigation.

\section{CONCLUSIONS}

The present study obtained a new finding in which the negative effects of family stigma on psychological well-being in women with infertility was due to self-stigma. This mechanism has not been explored in previous studies. The results suggest that women who struggle with infertility have worse emotional and self-esteem outcomes when they internalize family stigma. In clinical settings, it may be important for clinicians to evaluate the extent to which patients agree with infertility stigma, the reason why they agree with it, and any relevant automatic negative thoughts that may arise (e.g., the infertility is all my fault).

\section{LIMITATIONS}

The present study used a convenience sample at only one reproductive medical center at a private hospital 
in Taiwan. Hence, caution should be applied when interpreting or generalizing the results to other contexts. Whether there will be different patterns between different cultures needs further investigation. The sample was also limited in terms of gender. The literature suggests that men with infertility are also influenced by stigma, which means that it is also necessary to investigate the phenomenon among male partners in couples dealing with infertility. Also, to examine the influence of the stigma endorsed by others, the present study only measured family stigma. Future research can take Pescosolido and Martin's (2015) concept of multiple dimensions of public stigma into account. Lastly, the study used a cross-sectional design where the data were collected at a single point in time. Therefore, a clear causal relationship cannot be determined. Future research should investigate the relationship between infertility stigma and psychological well-being using a longitudinal design.

\section{REFERENCES}

Abbey, A., Andrews, F. M., \& Halrnan, L. J. (1991). Gender's role in responses to infertility. Psychology of Women Quarterly, 15, 295-316. https://doi. org/10.1111/j.1471-6402.1991.tb00798.x

Chang, H. C., \& Holt, G. R. (1991). More than relationship: Chinese interaction and the principle of kuan-hsi. Communication Quarterly, 39, 251-271. https://doi.org/10.1080/01463379109369802

Cheng, C. Y., Stevenson, E. L., Yang, C. T., \& Liou, S. R. (2018). Stress and quality of life for Taiwanese women who underwent infertility treatment. Journal of Obstetric, Gynecologic \& Neonatal Nursing, 47, 498-508.https://doi.org/10.1016/j.jogn.2018.03.005

Corrigan, P. W., Rafacz, J., \& Rüsch, N. (2011). Examining a progressive model of self-stigma and its impact on people with serious mental illness. Psychiatry Research, 189, 339-343. https://doi.org/ 10.1016/j.psychres.2011.05.024

Corrigan, P. W., \& Watson, A. C. (2002). The paradox of self-stigma and mental illness. Clinical Psychology: Science and Practice, 9, 35-53. https://doi.org/ 10.1093/clipsy.9.1.35

Donkor, E. S., \& Sandall, J. (2007). The impact of perceived stigma and mediating social factors on infertility-related stress among women seeking infertility treatment in Southern Ghana. Social Science \& Medicine, 65, 1683-1694. https://doi.org/ 10.1016/j.socscimed.2007.06.003

Else-Quest, N. M., LoConte, N. K., Schiller, J. H., \& Hyde, J. S. (2009). Perceived stigma, self-blame, and adjustment among lung, breast and prostate cancer patients. Psychology and Health, 24, 949964. https://doi.org/10.1080/08870440802074664

Ergin, R. N., Polat, A., Kars, B., Öztekin, D., Sofuoğlu, K., \& Çalışkan, E. (2018). Social stigma and familial attitudes related to infertility. Turkish Journal of Obstetrics and Gynecology, 15, 46-49. https://doi.org/10.4274/tjod.04307

Fu, B., Qin, N., Cheng, L., Tang, G., Cao, Y., Yan, C., Huang, X., Yan, P., Zhu, S., \& Lei, J. (2015). Development and validation of an infertility stigma scale for Chinese women. Journal of Psychosomatic Research, 79, 69-75. https://doi.org/10.1016/j.jpsychores.2014.11.014

Goffman, E. (1963). Stigma: Notes on the management of spoiled identity. Prentice-Hall.

Hsu, Y. L., \& Kuo, B. J. (2002). Evaluations of emotional reactions and coping behaviors as well as correlated factors for infertile couples receiving assisted reproductive technologies. The Journal of Nursing Research, 10, 291-302. https://doi.org/ 10.1097/01.jnr.0000347610.14166.52

Hu, L. T., \& Bentler, P. M. (1999). Cutoff criteria for fit indexes in covariance structure analysis: Conventional criteria versus new alternatives. Structural Equation Modeling: A Multidisciplinary Journal, 6, 1-55. https://doi.org/10.1080/10705519909540118

Huang, M. Z., Sun, Y. C., Gau, M. L., Puthussery, S., \& Kao, C. H. (2019). First-time mothers' experiences of pregnancy and birth following assisted reproductive technology treatment in Taiwan. Journal of Health, Population and Nutrition, 38, 1-11. https:// doi.org/10.1186/s41043-019-0167-3

Jin, X., Wang, G., Liu, S., Zhang, J., Zeng, F., Qiu, Y., \& Huang, X. (2013). Survey of the situation of infertile women seeking in vitro fertilization treatment in China. BioMed Research International, 2013, 179098. https://doi.org/10.1155/2013/179098

Jussim, L., Nelson, T. E., Manis, M., \& Soffin, S. (1995). Prejudice, stereotypes, and labeling effects: Sources of bias in person perception. Journal of Personality and Social Psychology, 68, 228-246. https:// doi.org/10.1037/0022-3514.68.2.228

Klemetti, R., Raitanen, J., Sihvo, S., Saarni, S., \& Koponen, P. (2010). Infertility, mental disorders and well-being-a nationwide survey. Acta Obstetricia et Gynecologica Scandinavica, 89, 677-682. https:// doi.org/10.3109/00016341003623746

Lau, J. T., Wang, Q., Cheng, Y., Kim, J. H., Yang, X., \& Yi Tsui, H. (2008). Infertility-related perceptions and responses and their associations with quality of life among rural Chinese infertile couples. Journal of Sex \& Marital Therapy, 34, 248-267. https:// doi.org/10.1080/00926230701866117

Lee, M. (2009). Transition to below replacement fertility and policy response in Taiwan. The Japanese Journal of Population, 7, 71-86.

$\mathrm{Li}, \mathrm{C}$. (2008). Production and influence of the Chinese tradition birth idea. Journal of North China Institute of Water Conservancy and Hydroelectric Power (Social Sciences Edition), 24, 77-80.

Lin, Y. N., Tsai, Y. C., \& Kung, C. Y. (2006). Taiwanese infertile women's experiences of infertility: a rela-
Infertility stigma in Taiwan 
Yi-Tung Lin, Ashley W. Wang, Shirley Wei, Jia-Sin Hong, Wen-Yau Hsu tional perspective. Chinese Annual Report of Guidance and Counseling, 19, 175-210.

Logan, S., Gu, R., Li, W., Xiao, S., \& Anazodo, A. (2019). Infertility in China: Culture, society and a need for fertility counselling. Asian Pacific Journal of Reproduction, 8, 1-6. https://doi.org/10.4103/23050500.250416

Loke, A. Y., Yu, P. L., \& Hayter, M. (2012). Experiences of sub-fertility among Chinese couples in Hong Kong: a qualitative study. Journal of Clinical Nursing, 21, 504-512. https://doi.org/10.1111/j.13652702.2010.03632.x

McNair, D. M., Lorr, M., \& Droppleman, L. F. (1971) Manual for the Profile of Mood States. Educational and Industrial Testing Services.

Pescosolido, B. A., \& Martin, J. K. (2015). The stigma complex. Annual Review of Sociology, 41, 87-116. https://doi.org/10.1146/annurev-soc-071312-145702

Poston, D. J. (1988). Childlessness patterns in Taiwan. Journal of Population Studies, 11, 55-78.

Rosenberg, M. (1965). Society and the adolescent selfimage. Princeton University Press.

Slade, P., O’Neill, C., Simpson, A. J., \& Lashen, H. (2007). The relationship between perceived stigma, disclosure patterns, support and distress in new attendees at an infertility clinic. Human Reproduction, 22, 2309-2317. https://doi.org/10.1093/humrep/dem 115

Stanton, A. L. (1991). Cognitive appraisals, coping processes, and adjustment to infertility. In A. L. Stanton \& C. Dunkel-Schetter (Eds.), Infertility (pp. 87-108). Springer.

Tiu, M. M., Hong, J. Y., Cheng, V. S., Kam, C. Y., \& Ng, B. T. (2018). Lived experience of infertility among Hong Kong Chinese women. International Journal of Qualitative Studies on Health and WellBeing, 13, 1554023. https://doi.org/10.1080/174826 31.2018.1554023

Tsui, A. S., \& Farh, J. L. L. (1997). Where guanxi matters: Relational demography and guanxi in the Chinese context. Work and Occupations, 24, 56-79. https://doi.org/10.1177/0730888497024001005

Vogel, D. L., Wade, N. G., \& Hackler, A. H. (2007). Perceived public stigma and the willingness to seek counseling: The mediating roles of self-stigma and attitudes toward counseling. Journal of Counseling Psychology, 54, 40-50. https://doi.org/10.1037/00220167.54.1.40

Wagner, J. A., III (1995). Studies of individualism-collectivism: Effects on cooperation in groups. Academy of Management Journal, 38, 152-173. https:// doi.org/10.5465/256731

World Population Review (2019). Taiwan population 2019. Retrieved from http://worldpopulationreview.com/countries/taiwan-population/ [accessed December 18, 2019]

$\mathrm{Wu}, \mathrm{C}$. L. (2002). Stigmatized gender and gendered stigma: The "infertile" men women in Taiwan. Tai- wanese Journal of Sociology, 29, 127-179. https:// doi.org/10.6786/TJS

Yang, Y. O., Peden-McAlpine, C., \& Chen, C. H. (2007). A qualitative study of the experiences of Taiwanese women having their first baby after the age of 35 years. Midwifery, 23, 343-349. https://doi. org/10.1016/j.midw.2006.03.009

Yao, H., Chan, C. H. Y., \& Chan, C. L. W. (2018). Childbearing importance: a qualitative study of women with infertility in China. Research in Nursing \& Health, 41, 69-77. https://doi.org/10.1002/ nur.21846

Yeh, K. H. (2003). The beneficial and harmful effects of filial piety: an integrative analysis. In K. S. Yang, K. K. Hwang, P. B. Pedersen, \& I. Daibo (Eds.), Progress in Asian social psychology: Conceptual and empirical contributions (pp. 67-82). Praeger Publishers/Greenwood Publishing Group. 